\title{
A Quality-Aware Relay Station Deployment Scheme for Green Radio Communication
}

\author{
M. Arthi ${ }^{1}$, P. Arulmozhivarman ${ }^{2}$ and K. Vinoth Babu ${ }^{1, *}$ \\ ${ }^{1}$ School of Electronics Engineering, VIT University, \\ Vellore, Tamil Nadu, India \\ ${ }^{2}$ School of Electrical Engineering, VIT University, \\ Vellore, Tamil Nadu, India \\ E-mail: $\{$ arthimdas; vinothbab\}@gmail.com; parulmozhivarman@vit.ac.in \\ ${ }^{*}$ Corresponding Author
}

Received 3 August 2016; Accepted 29 September 2016;

Publication 15 November 2016

\begin{abstract}
Power efficient transmission has received significant attention among cellular network operators to reduce the environmental effects and to maintain profitability. Since the evolved node base station (eNB) is the primary power consumer in the cellular network, endeavours have been made to study eNB power consumption and to find approaches to enhance power efficiency. The concept of multi-hop relay (MHR) network is introduced in $4 \mathrm{G}$ and beyond standards to reduce the deployment cost and transmission power while improving the coverage and capacity. Here relay stations (RS) are also deployed by the network operators along with eNBs to maximize the average spectral efficiency per user. But the deployment of RSs is not standardized. Improper RS deployment leads to severe quality loss. In this work, RS deployment based on cost (RDBC) approach is utilized which identifies the RS deployment combinations and deploys different types of RSs to maximize the system quality. Through simulations, it is validated that the proposed approach maximizes the transmission quality, coverage and effectiveness in power consumption and minimizes the deployment cost and interference when compared to many of the conventional RS deployment
\end{abstract}

Journal of Green Engineering, Vol. 6_2, 1-28.

doi: 10.13052/jge1904-4720.623

(c) 2016 River Publishers. All rights reserved. 
approaches. The scheme utilized ensures power efficient transmission and acts as a potential candidate for green radio communication.

Keywords: Green radio communication, Multi-hop relay (MHR) network, Power consumption effectiveness index (PCEI), RS deployment based on cost (RDBC), Relay Station (RS) deployment.

\section{Introduction}

The mobile users are increasing day by day. In 2020, the number of mobile subscribers (MS) is expected to be 7.6 billion [1]. The tremendous increase in the number of MSs and the broadband data requirement forces the operators to increase the number of macro eNBs. In telecommunication network 65-75\% of power is consumed by eNB sites. $4 \%$ of global $\mathrm{CO}_{2}$ emissions are from telecommunication industries $[2,3]$. Hence, the increase in the number of eNBs will lead to immense power consumption. It also increases the $\mathrm{CO}_{2}$ emissions in the environment. In addition to this, deployment and maintenance cost is also a major problem faced by the network operators. In cellular communication, service coverage and transmission quality are affected by interference, shadowing and Gaussian noise etc [4]. These effects may be reduced by deploying more number of eNBs. Deployment of more number of eNBs will increase the interference, power consumption and network cost. The increase in network cost increases the cost per bit, which is undesired from customer point of view.

It is observed that the MSs in the world consume 0.2-0.4 GW power annually [2, 3]. Similarly, the eNB sites in the world consume 4.5-9 MWh power annually. The high power consumption of MSs and eNBs have raised the financial and environmental concerns. The transmission power requirement of MSs increases with the increase in the data traffic volume. This reduces the life time of rechargeable batteries. This also increases the annually disposed batteries. The power consumption composition of a network operator is shown in Figure 1.

$151 \mathrm{MtCO}_{2}$ was emitted from the information and communication technology (ICT) industries during 2002. This is expected to reach $349 \mathrm{MtCO}_{2}$ in 2020. The $\mathrm{CO}_{2}$ emissions from ICT industries are expected to increase $6 \%$ every year. The yearly $\mathrm{CO}_{2}$ emissions from ICT industries are almost equivalent to the $\mathrm{CO}_{2}$ emissions from more than 8 million cars [5]. These large scale $\mathrm{CO}_{2}$ emissions will negatively influence the air and leads to climatic changes. This results in loss of eco system and increase in greenhouse effect. The eNB sites consist of transceiver, power amplifier, combiner, duplexer, 


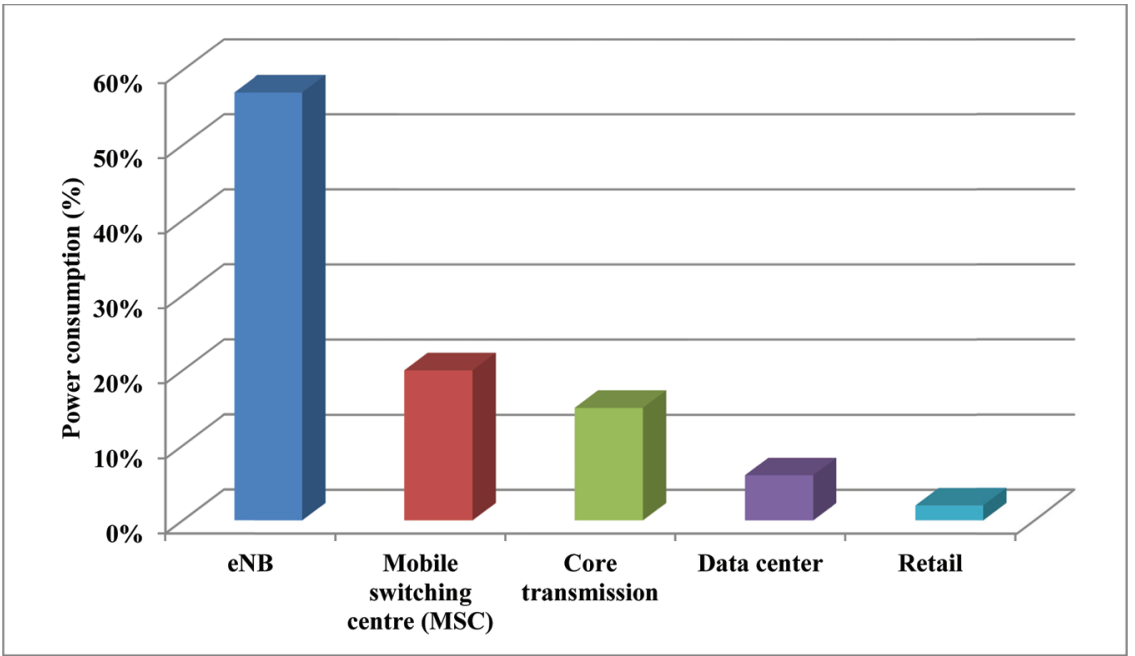

Figure 1 Power consumption composition of a network operator [1].

antenna, alarm extension system, control function, baseband receiver unit, cooling unit and generator. In this, about $60 \%$ of power is consumed by cooling units and generator. The increase in the number of eNB sites increases the carbon footprint. The distribution of power consumption at an eNB site is illustrated in Figure 2.

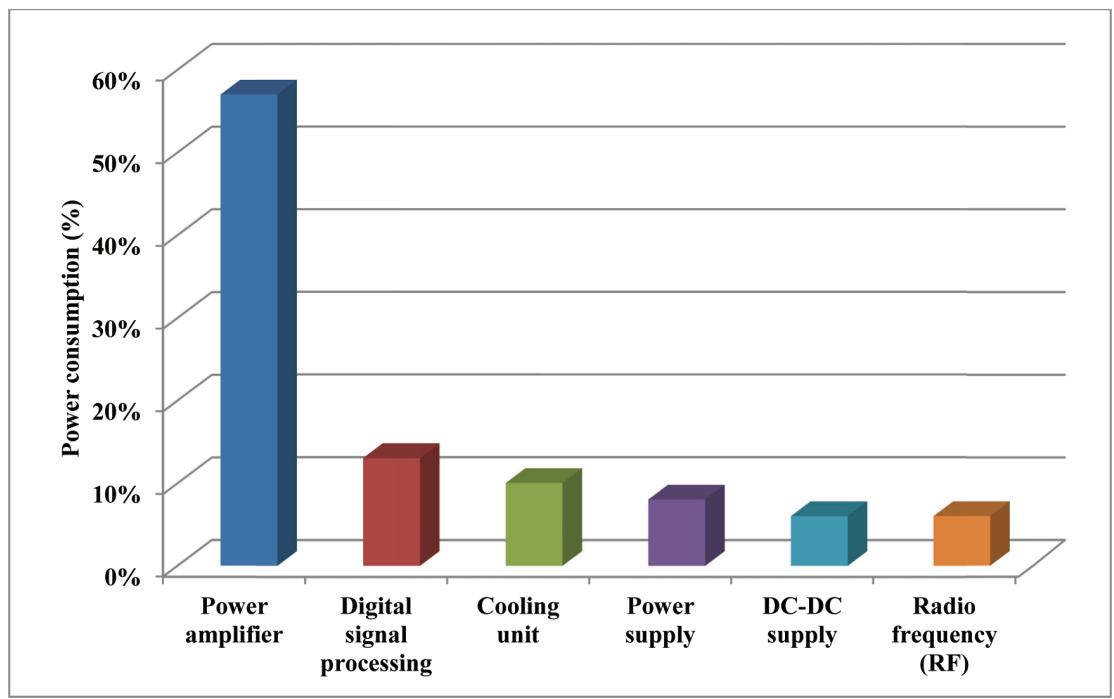

Figure 2 Distribution of power consumption at an eNB site [1]. 
In literature, there exist various solutions for green radio communication. Some of them are highlighted in this section. The power consumption of eNB can be minimized by using additional efficient software and hardware. An eNB consist of three major blocks like baseband, feeder and radio. The radio block consumes $80 \%$ of power out of which $50 \%$ is mainly due to the power amplifiers. In power amplifier, $80-90 \%$ of power is wasted as heat. The power consumption of the power amplifier also depends on the modulation type, frequency of operation and environment. The power consumption of power amplifier can be reduced by using the digital pre-distorted Doherty architecture power amplifier or AlGaN (Aluminium Gallium Nitride) based Power amplifier [6]. Switch mode power amplifiers also helps to reduce power consumption in eNB. The feeder cable power loss can be reduced by using suitable radio frequency (RF) heads and modular eNB design.

Due to user mobility, traffic load in each cell varies with time. The traffic load is more in office areas at day time and more in residential areas at night time. Due to this, some of the cells will be under low traffic load whereas other will be under high traffic load. Thus, a static cell deployment is inefficient to tackle imperfect load distributions. The ideas like cell zooming and cell breathing helps the cells to adjust their size depending on the traffic load conditions. These ideas improve the battery life and throughput. The eNBs with low traffic load are switched off periodically to consume power [7-11]. The usage of low power RF cables between the RF transmitter and antenna will also improve the power consumption. This actually keeps RF amplifier nearer to the antenna section than the conventional long coaxial cables.

The usage of power saving protocols such as discontinuous reception (DRX) and discontinuous transmission (DTX) modes also reduce the power consumption. The DRX and DTX momentarily power down the mobile devices to save power [1]. During transmission, power wastage occurs in antenna section due to reflections from the antenna back to the transceiver. These reflections are mainly due to the antenna environment, power level and impedance matching amplifier. These reflections can be cancelled by actively tuning the matching networks.

The techniques like multi input multi output (MIMO), channel coding, cognitive radio, power efficient scheduling are also used for power efficient transmission $[12,13]$. Instead of using conventional energy resources, renewable resources such as biofuels, wind energy, hydro and solar energy can be used in eNB to reduce power consumption [14]. With the usage of renewable resources in eNB, 6.8 million tons of $\mathrm{CO}_{2}$ emissions can be reduced per year. Similarly, 2.5 billion litres of diesel can be saved per year. 
The concept of MHR network is very popular in wireless standards like long term evolution-advanced (LTE-A) and worldwide interoperability for microwave access (WiMAX), where RSs are deployed by the network operators along with eNBs. The RSs replace the long distance communication link by many short distance communication links. This increases the link quality and transmission data rate [15]. RS deployment is useful in geographical areas, where backhaul connections are difficult and expensive [16]. For temporary deployments, RSs are more preferred than eNBs because of low cost and easy to carry nature. Since RS consumes less power than eNB, it is one of the best solutions for green radio communication [17]. The smaller physical dimension, low power and low cost features made RS more attractive in $4 \mathrm{G}$ and beyond cellular networks. To increase the coverage ratio and capacity, the deployment of RSs are entertained than the eNBs. The RS deployment sites do not require cooling units. It also involves less signal processing than eNB. Unfortunately, the deployment scheme for RSs is not standardized. An RS deployment scheme, which maximizes the signal strength quality and coverage while minimizing the deployment cost and interference, is required.

$\mathrm{Yu}, \mathrm{Y}$. et al. formulated a cost function for eNB and RS deployment. But this scheme does not consider the factors like interference and coverage [18]. Kim, S. J. et al. proposed a cost effective deployment scheme by considering sectored eNB and different network topologies. Here, the authors have not considered the concept of adaptive modulation and coding (AMC). They have also assumed a geographic area with uniform traffic demand [19]. Ge, Y. et al. proposed a cost effective RS deployment for mobile transport systems. But the effect of link overloading on the system performance is not considered [20]. Based on the K-supplier concepts, a joint base station (BS) and RS placement (JBRP) scheme is proposed in [21] for IEEE $802.16 \mathrm{j}$ networks. This scheme maximizes the system throughput by maintaining lower deployment cost. But this scheme suffers by network load imbalance between eNBs. This load imbalance leads to decreased network throughput and increased packet queuing delay.

Chang, J. Y. and Chen, Y. W. have proposed an RS selection and deployment scheme based on uniform clustering algorithm [22]. It is showed that the coverage and throughput performance of this scheme is superior to the JBRP scheme. Vincent, M. et al. proposed a fuzzy logic based RS selection and deployment scheme for IEEE $802.16 \mathrm{j}$ networks [23]. The coverage ratio and traffic ratio of each candidate site is given as the input for fuzzy inference engine. Based on the fuzzy output parameter selection factor, the RS 
candidate positions are selected. All the above RS deployment schemes are developed for maximizing the coverage and capacity. They failed to consider the performance degradation due to inter RS interference. The deployment of different types of RSs is also not addressed in the above works.

Chang, B. J. et al. proposed an adaptive cost based RS deployment (ACRD) scheme by considering different impact parameters like signal strength quality, deployment cost, coverage ratio with and without population and RS overlapping index [4]. The authors developed a cost function based on these impact parameters. This scheme basically addresses the inter RS interference and the deployment of different types of RS. Through simulation results, it is proved that ACRD scheme offers better performance than random deployment (RAND) [20], static average weighting (SWA) [24] and dynamic average weighting (DWA) [25] schemes. But this scheme failed to address the green communication aspects and power consumption.

The power transmitted from the RSs must be effectively used by the MSs. Hence, the RSs must be effectively deployed in suitable candidate sites such that the power transmitted reaches the MSs without much path loss. In order to avoid the wastage of power, maximizing the power consumption effectiveness is set as one of the objectives of this work. In the proposed scheme, a network cost function is formulated by considering five different impact factors like signal strength quality index, deployment cost index, coverage ratio index, RS overlapping index and power consumption effectiveness index. This problem is solved for least network cost.

The rest of the manuscript is organized in the following order: The system model is described in Section 2. The proposed multi-objective RS deployment scheme is briefed in Section 3. The simulation results are discussed in Section 4 and the manuscript is concluded in Section 5.

\section{System Model}

The concept of MHR network is very popular in 4G and beyond standards, which provides a high quality continuous connection to the MSs anywhere in the geographical area. The idea of transparent (Type II) and non-transparent (Type I) RSs is defined in LTE-A and IEEE 802.16 j standards, which are also compared in Table 1.

Type I RSs are usually deployed on the cell edges. It acts like a separate serving node. It operates in half duplex mode and has its own physical ID, reference symbols, and synchronization channels. Type II RSs are usually 
Table 1 Transparent vs. Non-transparent RS

\begin{tabular}{lll}
\hline & Transparent RS (Type II) & Non-Transparent RS (Type I) \\
\hline Cost & Low & High \\
Number of Hops & 2 & 2 or more \\
Coverage extension & No & Yes \\
Scheduling & Centralized & Distributed \\
Performance & High & Low \\
\hline
\end{tabular}

deployed within the cell coverage, where there is serious signal attenuation due to shadowing and other propagation effects. eNB generates the control and reference signals for these types of RSs. Based on the mobility support, RSs are classified into three different types namely Fixed RS (FRS), Nomadic RS (NRS) and Mobile RS (MRS). MHR network with different types of RSs are illustrated in Figure 3. The coverage area of FRS is more than NRS and MRS. FRS can operate in line of sight (LOS) and non-line of sight (NLOS) conditions. FRSs are usually mounted in the areas where high signal attenuation is present. NRSs are more suited for temporary deployments. It is usually deployed in the areas, where more number of users will be present for short period of time. MRSs are usually mounted on the public transportation systems, which provide continuous connection to the moving users. The different impact parameters used in this work are explained below in detail.

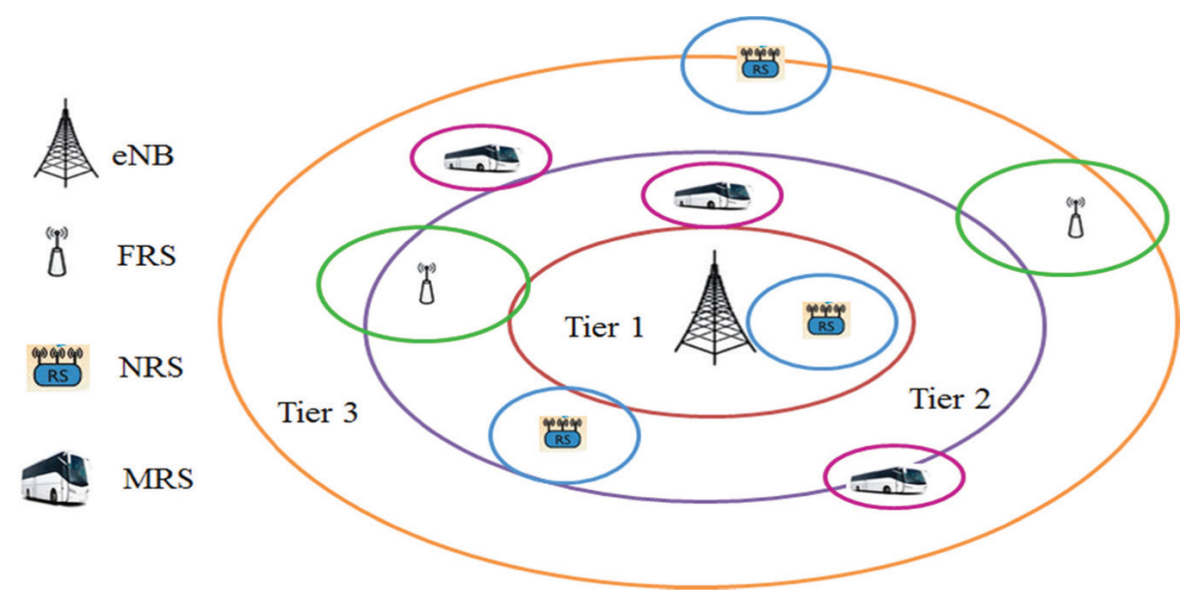

Figure 3 MHR network with different types of RSs. 


\subsection{Signal Strength Index (SSI)}

SSI represents the transmission signal quality, which depends on the signal to noise ratio (SNR) experienced by each user. The concept of AMC is also very popular in $4 \mathrm{G}$ and beyond standards, which maximizes the spectral efficiency per user [26, 27]. Here, each eNB coverage range is divided into different tiers. The eNB allocates different modulation, codes and code rates to users in different tiers. The users in the cell edge tiers experience reduced data rate than the users in other tiers. The efficient deployment of different types of RSs reduces the distance between the serving node and MS. The introduction of RSs will reduce the transmission power requirement and increase the throughput per user. The parameter SSI is given by

$$
S S I=\sum_{i=1}^{T} \sum_{r=1}^{R} \sum_{j=1}^{N_{r}^{i}} D R^{j}
$$

where $T$ and $R$ represents the number of tiers and the number of RS types respectively. $N_{r}^{i}$ denotes the number of $r^{\text {th }}$ type of RS in $i^{\text {th }}$ tier and $D R^{j}$ represents the average data transmission rate of $j^{\text {th }} \mathrm{RS}$. This parameter does not depend on the type of RS rather it depends on the location of RS. A good RS deployment combination should maximize SSI.

\subsection{RS Deployment Price Index (RPI)}

The metric RPI is used to find the RS deployment cost in the given geographical area. This parameter does not depend on the location rather it depends on the type of RS. The cost of FRS is more than the other types of RSs. The parameter $\mathrm{RPI}$ is given by

$$
R P I=\sum_{i=1}^{T} \sum_{r=1}^{R}\left(N_{r}^{i} \cdot D C_{r}\right)
$$

where $D C_{r}$ represents the deployment cost of $r^{\text {th }}$ type of RS. Since this parameter is directly related to the operator deployment cost and cost per bit, it should be minimized. This parameter can be minimized by minimizing the number of FRS deployments.

\subsection{Coverage Index $(\mathrm{Cl})$}

One of the major objectives of RS deployment is to increase the service coverage. An effective RS deployment combination should maximize CI by minimizing the coverage holes. The parameter $\mathrm{CI}$ is given by 


$$
C I=\frac{\sum_{i=1}^{T} \sum_{r=1}^{R} \sum_{j=1}^{N_{r}^{i}} K^{j}}{N}
$$

where $K^{j}$ denotes the number of MSs covered by $j^{\text {th }}$ RS and $N$ represents the number of MSs in the geographic area.

\subsection{RS Interference Index (RII)}

When several RSs transmit their signals on the same frequency in the same geographic area, the MSs may not be able to distinguish to which RS it is listening. The imperfect cell structures leads to severe inter cell interference at the cell edges. The interference reduces the link quality of cell edge MSs. This degrades the user experiencing data rate and increases the outages. The interference situation is unpredictable and more severe in MHR networks. There exist two possible types of interference in MHR networks. The co-tier interference occurs between the MSs of the different RSs or eNBs and the cross-tier interference occurs between MSs of the eNBs and RSs. The cross-tier interference is difficult to control.

Sometimes RSs are expected to serve users even at low signal to interference plus noise ratio (SINR), which requires a strong mechanism to minimize or cope with interference. The inter cell interference control (ICIC) feature is proposed in LTE Rel-10 with the specifications of high interference indication (HII), overload indication and relative narrowband transmit power indicator (RNTP) signalling between eNBs. But this feature cannot deal with the interference to/from reference signals and control channels. In contrast, RSs that are adequately far from each other may reuse the same frequency bands with less interference. Hence, in this work, to minimize the co-tier and crosstier interferences, the RSs are placed adequately far from each other. This minimizes the interference between the RSs and maintains the link quality even at the cell edges. Many of the conventional MHR deployment schemes have not considered the interference minimization problem. In this work, we are addressing the interference problem in the RS deployment stage itself. This enables the reuse of radio resources on different RSs, which improves the system capacity.

This parameter RII depends on the overlapping area between different types of RSs. The increase in the overlapping area increases the inter RS interference. An effective RS deployment combination should minimize RII. When the number of RSs present in the geographical area increases, RII also increases, which leads to significant inter RS interference. The parameter RII of $j^{\text {th }} \mathrm{RS}$ is given by [28] 


$$
\begin{array}{r}
\left(b^{j}\right)^{2} \cos ^{-1}\left(\frac{\delta^{2}+\left(b^{j}\right)^{2}-a^{2}}{2 \delta b^{j}}\right)+a^{2} \cos ^{-1}\left(\frac{\delta^{2}+a^{2}-\left(b^{j}\right)^{2}}{2 \delta a}\right) \\
R I I^{j}=\frac{-\frac{1}{2} \sqrt{\left(-\delta+b^{j}+a\right)\left(\delta+b^{j}-a\right)\left(\delta-b^{j}+a\right)\left(\delta+b^{j}+a\right)}}{\pi\left(b^{j}\right)^{2}}
\end{array}
$$

where $b^{j}$ and $a$ denote the radius of $j^{\text {th }}$ RS and the radius of the neighbouring overlapped RS respectively. $\delta$ denotes the distance between the centre point of the two overlapped RSs respectively.

\subsection{Power Consumption Effectiveness Index (PCEI)}

The power transmitted from the serving RSs must be effectively used by the MSs. Hence, the RS deployment combinations should be optimally selected so that the power transmitted reaches the MSs without much path loss. In order to avoid power wastage, maximizing the parameter PCEI is set as one of the objectives of this work. The parameter PCEI of $j^{\text {th }} \mathrm{RS}$ is given by

$$
P C E I^{j}=\frac{\sum_{m=1}^{K} P^{j, m}}{K^{j} P_{t}^{j}}
$$

where $P^{j, m}$ represent the average received power of $m^{\text {th }} \mathrm{MS}$ from $j^{\text {th }} \mathrm{RS}$ and $P_{t}^{j}$ represent the average transmit power of $j^{\text {th }} \mathrm{RS}$. The total PCEI is given by

$$
\text { PCEI }=\sum_{i=1}^{T} \sum_{r=1}^{R} \sum_{j=1}^{N_{r}^{i}} P C E I^{j}
$$

\section{The Proposed RDBC Scheme}

In the proposed algorithm, a network cost function is formulated by considering five different impact parameters. The problem is solved for least network cost. The objectives of the proposed algorithm are

$$
\begin{aligned}
& \max \left\{S S I_{l}, C I_{l}, P C E I_{l}\right\}, \quad \forall l \in P \\
& \min \left\{R P I_{l}, R I I_{l}\right\}, \quad \forall l \in P
\end{aligned}
$$

where $P$ is the total number of RS deployment combinations. The proposed algorithm is executed in 6 steps. In the first step, the impact factors corresponding to every RS deployment combination is identified. These calculations 
are based on the Equations (1) to (6). In this multi-objective approach, some of these impact parameters should be maximized and some of them should be minimized. In Step 2, all these impact parameters are transferred to consistent parameters, so that when all these parameters decrease, the overall system performance increases. This is done using Equations (9) to (13). In the third step, all these consistent parameters are normalized with respect to $S S I^{*}$. This is done using Equations (14) to (18). In the fourth step, dynamic weights corresponding to each impact parameter is identified using Equations (19) to (24). A network cost function is formulated in Step 5. For every deployment combination, the network cost is identified using (25). In Step 6, a deployment combination corresponding to least network cost is identified using (26). Based on the identified locations and RS types, RSs are deployed.

Step 1: Identifying the impact parameters

$$
\begin{aligned}
& \text { for } l=1: P \\
& S S I=0, R P I=0, \quad C I=0, \quad R I I=0, P C E I=0 \\
& \text { for } i=1: T \\
& \text { for } r=1: R \\
& R P I=R P I+N_{r}^{i} \cdot D C_{r} \\
& \text { for } j=1: N_{r}^{i} \\
& C I=C I+\frac{K^{j}}{N} \\
& S S I=S S I+D R^{j} \\
& R I I=R I I+R I I^{j} \\
& \text { for } m=1: K^{j} \\
& P C E I=P C E I+\frac{p^{j, m}}{K^{j} p_{t}^{j}} \\
& \text { end for } \\
& \text { end for } \\
& \text { end for } \\
& \text { end for }
\end{aligned}
$$




\section{M. Arthi et al.}

Step 2: The impact parameters are transformed to consistent parameters

The impact factors SSI, CI and PCEI should be maximized and RPI and $R I I$ should be minimized. In order to form a network cost function, all these impact factors should be higher or lower for better result. SSI, CI and PCEI are transferred to new consistent parameters $\frac{1}{S S I}, \frac{1}{C I}, \frac{1}{P C E I}$. Now, the lesser value of every parameter yields a better result.

$$
\begin{aligned}
S S I^{*} & =\frac{1}{S S I} \\
R P I^{*} & =R P I \\
C I^{*} & =\frac{1}{C I} \\
R I I^{*} & =R I I \\
P C E I^{*} & =\frac{1}{P C E I}
\end{aligned}
$$

Step 3: Identifying the normalized weights for all impact parameters

The value ranges of different consistent impact factors are different. In order to make a common value range, every parameter is normalized to $S S I^{*}$.

$$
\begin{aligned}
W_{S S I^{*}} & =\frac{S S I^{*}}{S S I^{*}} \\
W_{R P I^{*}} & =\frac{S S I^{*}}{R P I^{*}} \\
W_{C I^{*}} & =\frac{S S I^{*}}{C I^{*}} \\
W_{R I I^{*}} & =\frac{S S I^{*}}{R I I^{*}} \\
W_{P C E I^{*}} & =\frac{S S I^{*}}{P C E I^{*}}
\end{aligned}
$$

Step 4: Identifying the dynamic weights for all impact parameters

Based on the previous studies, it is observed that fixed weighting and unfair weighting among various consistent impact factors have not given optimal results [4]. Hence, based on the principle of proportional formula, dynamic weights are computed for all the parameters.

$$
W_{T}=\sum_{q=1}^{I} W_{q}
$$


where $I$ is the number of impact parameters.

$$
\begin{aligned}
X_{S S I^{*}} & =\frac{W_{S S I^{*}}}{W_{T}} \\
X_{R P I^{*}} & =\frac{W_{R P I^{*}}}{W_{T}} \\
X_{C I^{*}} & =\frac{W_{C I^{*}}}{W_{T}} \\
X_{R I I^{*}} & =\frac{W_{R I I^{*}}}{W_{T}} \\
X_{P C E I^{*}} & =\frac{W_{P C E I^{*}}}{W_{T}}
\end{aligned}
$$

Step 5: Formulating the network cost function

The network cost function is formulated as the function of different consistent impact factors weighted by dynamic weights.

$$
\begin{aligned}
C_{l}= & X_{S S I^{*}} S S I^{*}+X_{R P I^{*}} R P I^{*}+X_{C I^{*}} C I^{*}+X_{R I I^{*}} R I I^{*} \\
& +X_{P C E I^{*}} P C E I^{*}
\end{aligned}
$$

\section{end for}

Step 6: Identifying the combination corresponding to the least network cost

The number of RSs in each tier and their types are decided based on the capacity of each tier. For every eNB capacity, the number of RS combinations is decided. For all the deployment combination, the proposed scheme is executed. The deployment combination with minimum network cost is identified for every eNB capacity.

$$
\text { Choose } l \leftarrow \min \left[C_{l}\right], \forall l \in P
$$

\section{Simulation Results and Discussions}

The performance of the proposed RS deployment scheme is validated through simulations using Matlab 2015a tool. The performance of the proposed scheme is compared with the conventional uniform clustering, RAND and fuzzy logic based RS deployment schemes. The parameters considered for the simulation study are listed in Table 2. For simulations, quadrature 


\section{M. Arthi et al.}

Table 2 Simulation settings

\begin{tabular}{|c|c|}
\hline \multicolumn{2}{|c|}{ Network Layout Settings } \\
\hline Size of the geographical area & $3 \mathrm{~km} \times 3 \mathrm{~km}$ \\
\hline Network elements & eNB, RS and MS \\
\hline \multicolumn{2}{|r|}{ eNB Settings } \\
\hline Wireless range & $3 \mathrm{~km}$ \\
\hline Capacity (Mbps) & $30-150$ \\
\hline Number of tiers & 3 \\
\hline \multirow{3}{*}{$\begin{array}{l}\text { Modulation schemes supported } \\
\text { in each tier }\end{array}$} & Tier 1:64-QAM \\
\hline & Tier 2:16-QAM \\
\hline & Tier 3:BPSK \\
\hline \multirow[t]{3}{*}{ Capacity of each tier (Mbps) } & Tier $1: 15$ \\
\hline & Tier 2:9 \\
\hline & Tier 3:6 \\
\hline \multicolumn{2}{|r|}{ RS Settings } \\
\hline \multirow[t]{3}{*}{ Wireless range (meters) } & FRS:375 \\
\hline & NRS:300 \\
\hline & MRS:225 \\
\hline \multirow[t]{3}{*}{ Deployment cost (units) } & FRS:1 \\
\hline & NRS:0.75 \\
\hline & MRS:0.5 \\
\hline \multicolumn{2}{|r|}{ MS Settings } \\
\hline Number of MSs & $300-500$ \\
\hline Population distribution & Population distribution \\
\hline \multicolumn{2}{|c|}{ Channel Settings } \\
\hline \multirow[t]{7}{*}{ Path loss model } & \multirow{2}{*}{$\begin{array}{l}\text { Log distance model }[11] \\
\text { The path loss in } \mathrm{dB} \text { for macro eNB is given } \\
\text { by }[29,30]\end{array}$} \\
\hline & \\
\hline & $\mathrm{PL}_{\mathrm{eNB}}(\mathrm{dB})=128.1+37.6 \log _{10}(\mathrm{~d})$ \\
\hline & \multirow{2}{*}{$\begin{array}{l}\text { where } \mathrm{d} \text { is the distance between eNB and MS } \\
\text { or RS. } \\
\text { Similarly, the path loss in } \mathrm{dB} \text { for RS is given } \\
\text { by [31] }\end{array}$} \\
\hline & \\
\hline & $\mathrm{PL}_{\mathrm{RS}}(\mathrm{dB})=103.8+20.9 \log _{10}(\mathrm{v})$ \\
\hline & where $\mathrm{v}$ is the distance between RS and MS. \\
\hline Mobility model for NRS and MRS & Random way point \\
\hline Velocities of NRS and MRS (kmph) & $10-100$ \\
\hline $\begin{array}{l}\text { Additive white Gaussian noise } \\
\text { variance }(\mathrm{dBm} / \mathrm{Hz})\end{array}$ & -174 \\
\hline
\end{tabular}


amplitude modulation (QAM) and binary phase shift keying (BPSK) modulations are considered. Some of the sample simulation scenarios are shown in Figures 4 to 7 for the eNB capacity of 90 Mbps. For the eNB capacity of $90 \mathrm{Mbps}$, we can formulate the RS combinations as shown in Tables 3 to 6. Out of these 4 combinations, one is chosen based on the least network cost. We can formulate many such combinations for every eNB capacity. The simulations are repeated for 100 different MS distributions and the average values of the impact parameters are alone displayed in this work.

Through simulations, it is validated that the system performance increases with the increase in eNB capacity irrespective of the RS deployment scheme. Figure 8 compares the minimum network cost of various deployment schemes for different values of eNB capacity. RDBC scheme yields the minimum network cost when compared to other schemes. Similarly, RAND scheme yields the worst network cost. The minimum network cost performance of the fuzzy logic based scheme is superior to uniform clustering scheme and inferior to RDBC scheme. All the other schemes except RDBC, always deploys FRSs even for the areas with very less population. For an eNB capacity of $150 \mathrm{Mbps}$, our proposed RDBC scheme offers an improvement of approximately 50\% over the conventional RAND scheme in terms of minimum network cost.

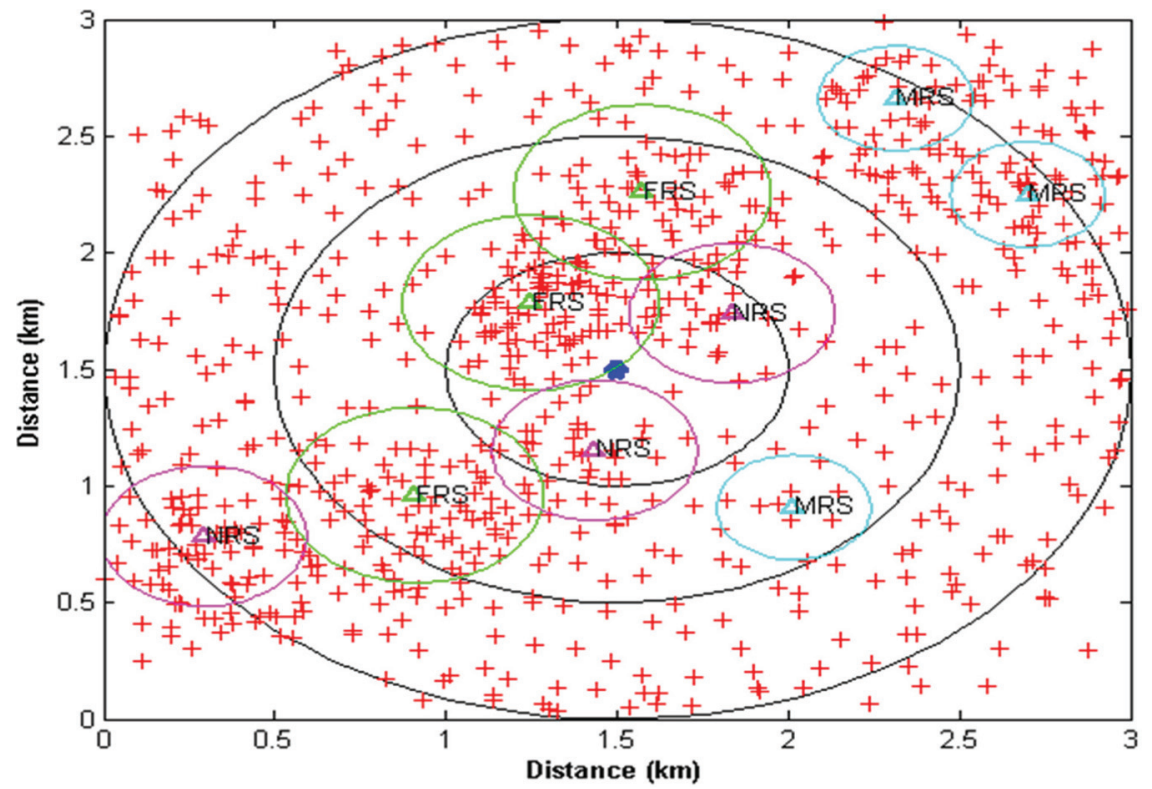

Figure 4 Sample scenario 1: RS deployment combinations for an eNB capacity of $90 \mathrm{Mbps}$. 


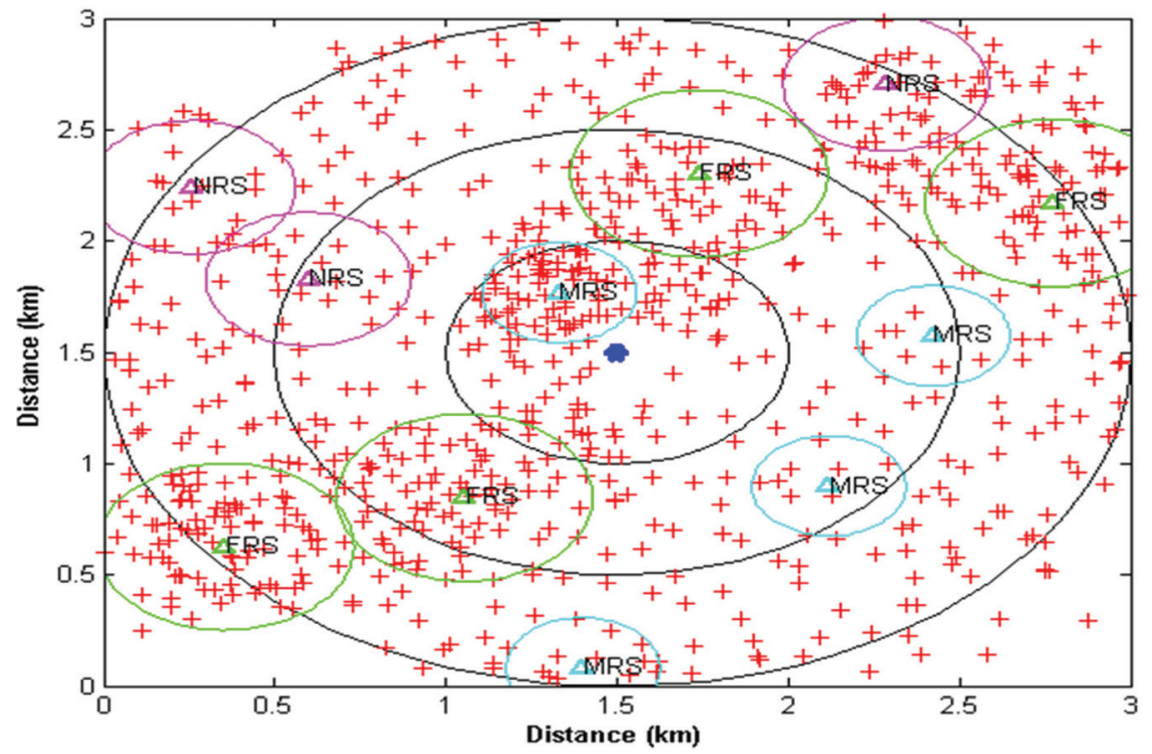

Figure 5 Sample scenario 2: RS deployment combinations for an eNB capacity of 90 Mbps.

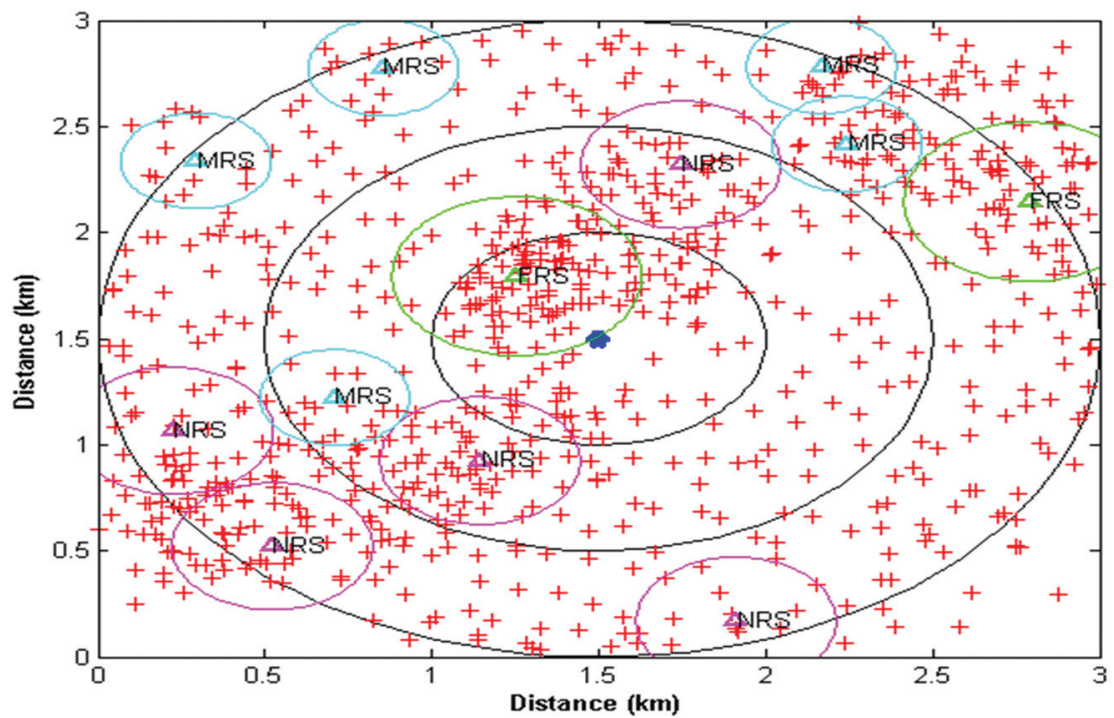

Figure 6 Sample scenario 3: RS deployment combinations for an eNB capacity of 90 Mbps. 


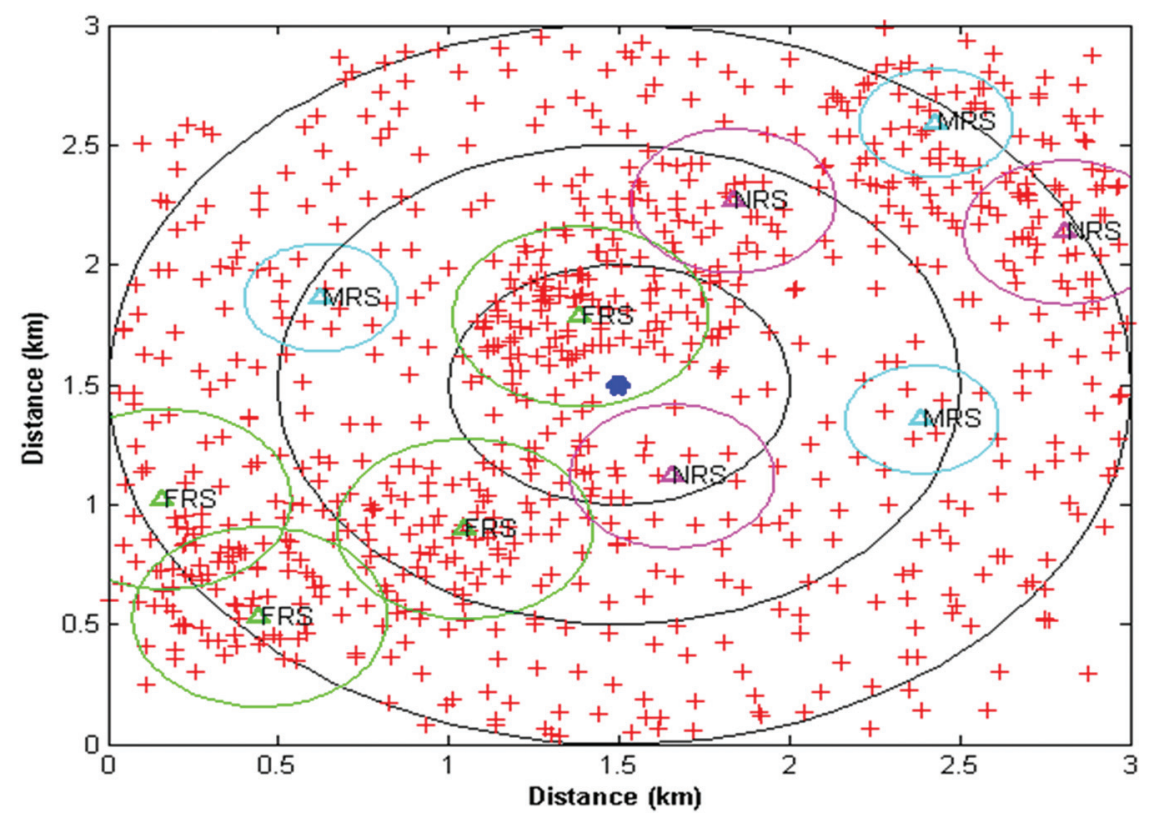

Figure 7 Sample scenario 4: RS deployment combinations for an eNB capacity of $90 \mathrm{Mbps}$.

Table 3 RS deployment combination 1 for the eNB capacity of $90 \mathrm{Mbps}$

\begin{tabular}{lccc}
\hline Type of RSs & $\begin{array}{c}\text { Number of RSs } \\
\text { in Tier 1 }\end{array}$ & $\begin{array}{c}\text { Number of RSs } \\
\text { in Tier 2 }\end{array}$ & $\begin{array}{c}\text { Number of RSs } \\
\text { in Tier 3 }\end{array}$ \\
\hline FRS & 1 & 2 & 0 \\
NRS & 2 & 0 & 1 \\
MRS & 0 & 1 & 2 \\
& $3 \times 15=45$ & $3 \times 9=27$ & $3 \times 6=18$ \\
& & & $90 \mathrm{Mbps}$ \\
\hline
\end{tabular}

Table 4 RS deployment combination 2 for the eNB capacity of 90 Mbps

\begin{tabular}{lccc}
\hline Type of RSs & $\begin{array}{c}\text { Number of RSs } \\
\text { in Tier 1 }\end{array}$ & $\begin{array}{c}\text { Number of RSs } \\
\text { in Tier 2 }\end{array}$ & $\begin{array}{c}\text { Number of RSs } \\
\text { in Tier 3 }\end{array}$ \\
\hline FRS & 0 & 2 & 2 \\
NRS & 0 & 1 & 2 \\
MRS & 1 & 2 & 1 \\
& $1 \times 15=15$ & $5 \times 9=45$ & $5 \times 6=30$ \\
& & & $90 \mathrm{Mbps}$ \\
\hline
\end{tabular}


Table 5 RS deployment combination 3 for the eNB capacity of $90 \mathrm{Mbps}$

\begin{tabular}{lccc}
\hline Type of RSs & $\begin{array}{c}\text { Number of RSs } \\
\text { in Tier 1 }\end{array}$ & $\begin{array}{c}\text { Number of RSs } \\
\text { in Tier 2 }\end{array}$ & $\begin{array}{c}\text { Number of RSs } \\
\text { in Tier 3 }\end{array}$ \\
\hline FRS & 1 & 0 & 1 \\
NRS & 0 & 2 & 3 \\
MRS & 0 & 1 & 4 \\
& $1 \times 15=15$ & $3 \times 9=27$ & $8 \times 6=48$ \\
& & & $90 \mathrm{Mbps}$ \\
\hline
\end{tabular}

Table 6 RS deployment combination 4 for the eNB capacity of $90 \mathrm{Mbps}$

\begin{tabular}{lccc}
\hline Type of RSs & $\begin{array}{c}\text { Number of RSs } \\
\text { in Tier 1 }\end{array}$ & $\begin{array}{c}\text { Number of RSs } \\
\text { in Tier 2 }\end{array}$ & $\begin{array}{c}\text { Number of RSs } \\
\text { in Tier 3 }\end{array}$ \\
\hline FRS & 1 & 1 & 2 \\
NRS & 1 & 1 & 1 \\
MRS & 0 & 2 & 1 \\
& $2 \times 15=30$ & $4 \times 9=36$ & $4 \times 6=24$ \\
& & $90 \mathrm{Mbps}$ \\
\hline
\end{tabular}

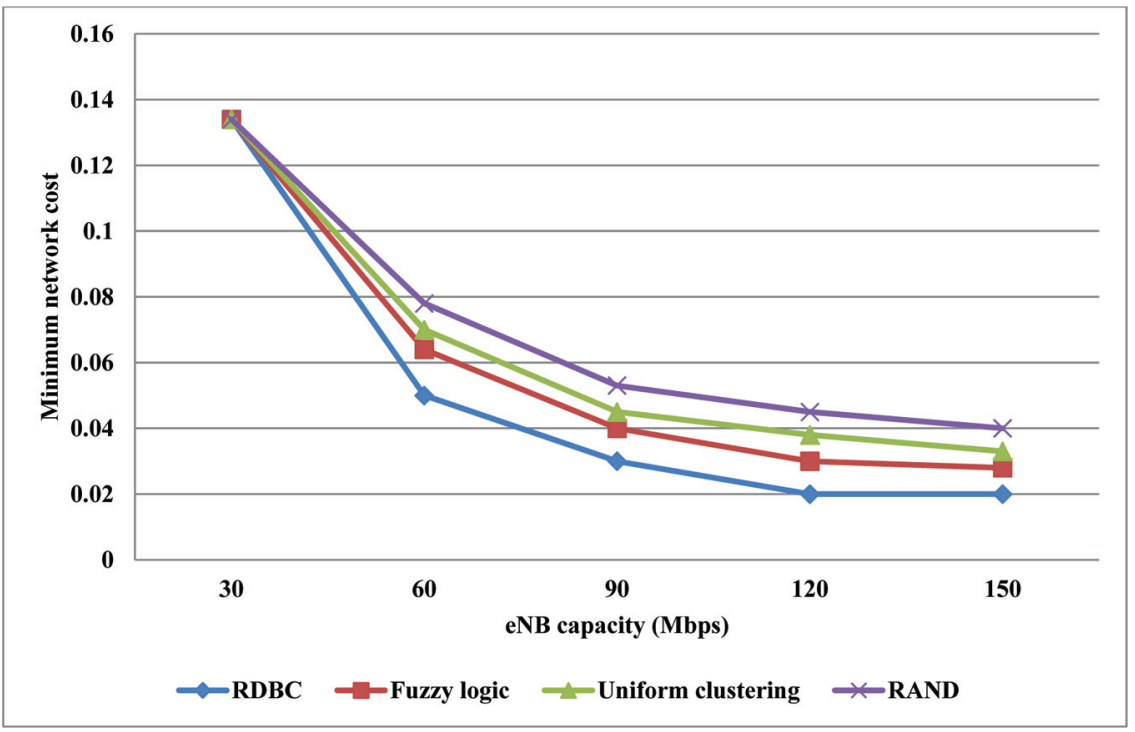

Figure 8 eNB capacity (Mbps) vs. Minimum network cost comparison for different RS deployment schemes.

Figure 9 compares the SSI impact factor of various deployment schemes for different values of eNB capacity. SSI increases with eNB capacity irrespective of the deployment scheme. RDBC scheme yields the maximum SSI 


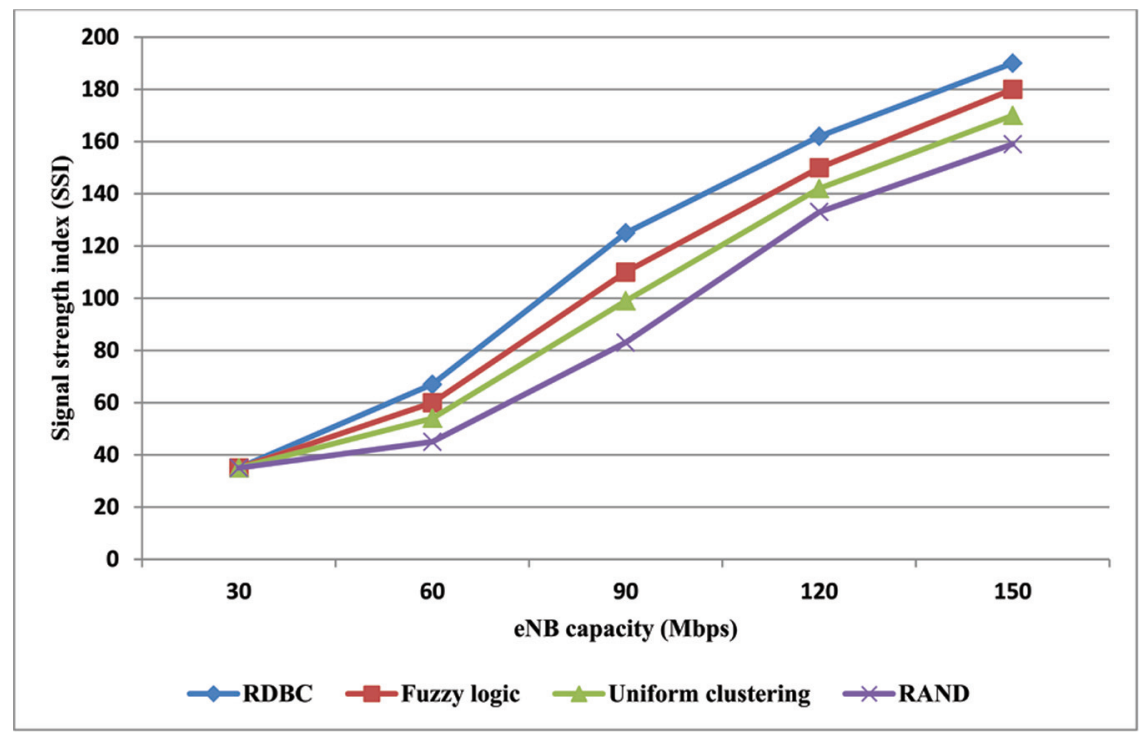

Figure 9 eNB capacity (Mbps) vs. SSI comparison for different RS deployment schemes.

when compared to other schemes. Similarly, RAND scheme yields the worst SSI. The SSI performance of the fuzzy logic based scheme is superior to uniform clustering scheme and inferior to RDBC scheme. In order to increase the signal strength quality, RDBC scheme deploys more number of FRSs in the high density populated areas. This increases the SSI. For an eNB capacity of 150 Mbps, our proposed RDBC scheme offers an improvement of approximately $16 \%$ over the conventional RAND scheme in terms of SSI.

Figure 10 compares the RPI impact factor of various deployment schemes for different values of eNB capacity. RPI increases with eNB capacity irrespective of the deployment scheme. The fuzzy logic based deployment scheme offers low RPI when compared to other schemes. The fuzzy logic based scheme deploys less number of FRSs than the other schemes. For most of the cases, our proposed RDBC scheme offers low RPI when compared with RAND scheme. To maximize the transmission signal quality, our proposed RDBC scheme deploys more number of FRSs than the other types of RSs. As mentioned in Table 2, the cost of FRS is larger than NRS and MRS. This increases the overall deployment cost. Our proposed RDBC scheme offers an average improvement of approximately $7 \%$ over the conventional RAND scheme in terms of RPI. 


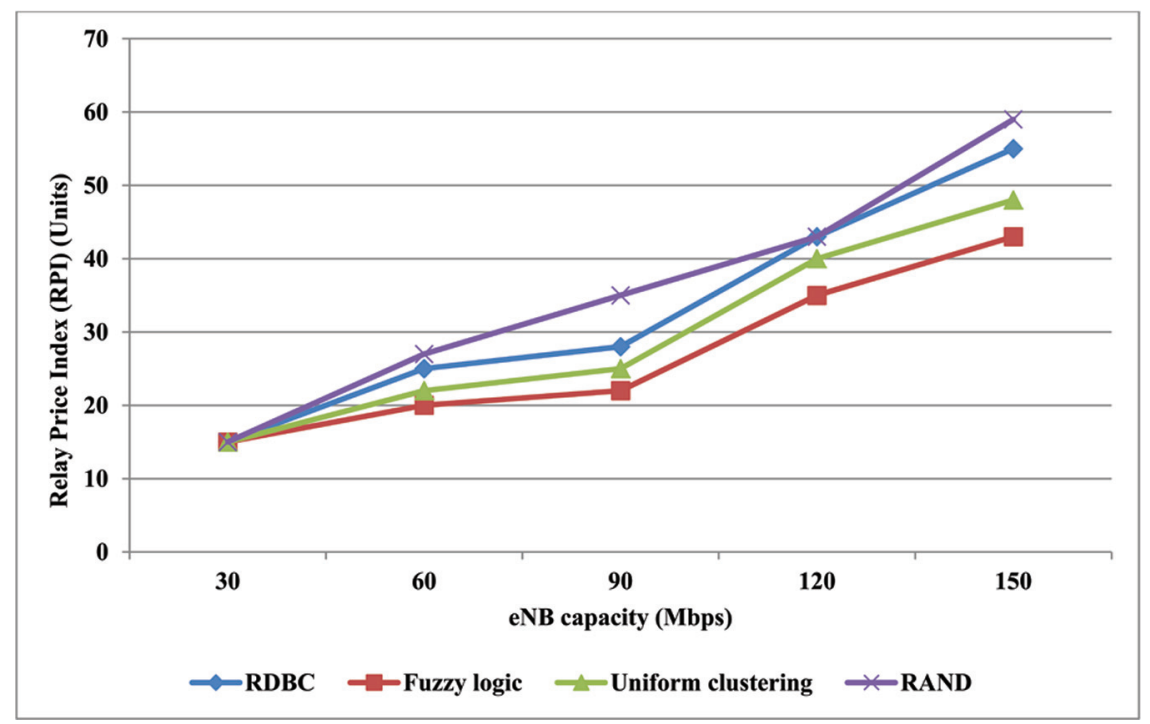

Figure 10 eNB capacity (Mbps) vs. RPI comparison for different RS deployment schemes.

Figure 11 compares the CI impact factor of various deployment schemes for different values of eNB capacity. CI increases with eNB capacity irrespective of the deployment scheme. The increase in eNB capacity, increases the number of deployed RSs. This increases the number of covered MSs. RDBC scheme yields the maximum CI when compared to other schemes. Similarly, RAND scheme yields the worst CI. The CI performance of the fuzzy logic based scheme is superior to uniform clustering scheme and inferior to RDBC scheme. In order to increase the coverage range, RDBC scheme deploys more number of FRSs in the high density populated areas. As mentioned in Table 2, the wireless range of FRS is larger than NRS and MRS. This covers more number of MSs and increases the CI. For an eNB capacity of $150 \mathrm{Mbps}$, our proposed RDBC scheme offers an improvement of approximately $26 \%$ over the conventional RAND scheme in terms of CI.

Figure 12 compares the RII impact factor of various deployment schemes for different values of eNB capacity. RII increases with eNB capacity irrespective of the deployment scheme. The increase in eNB capacity, increases the number of deployed RSs. This increases the overlapping area which in turn increases the inter RS interference. RDBC scheme yields the minimum RII when compared to other schemes. Similarly, RAND scheme yields the worst RII. The RII performance of the fuzzy logic based scheme is superior 


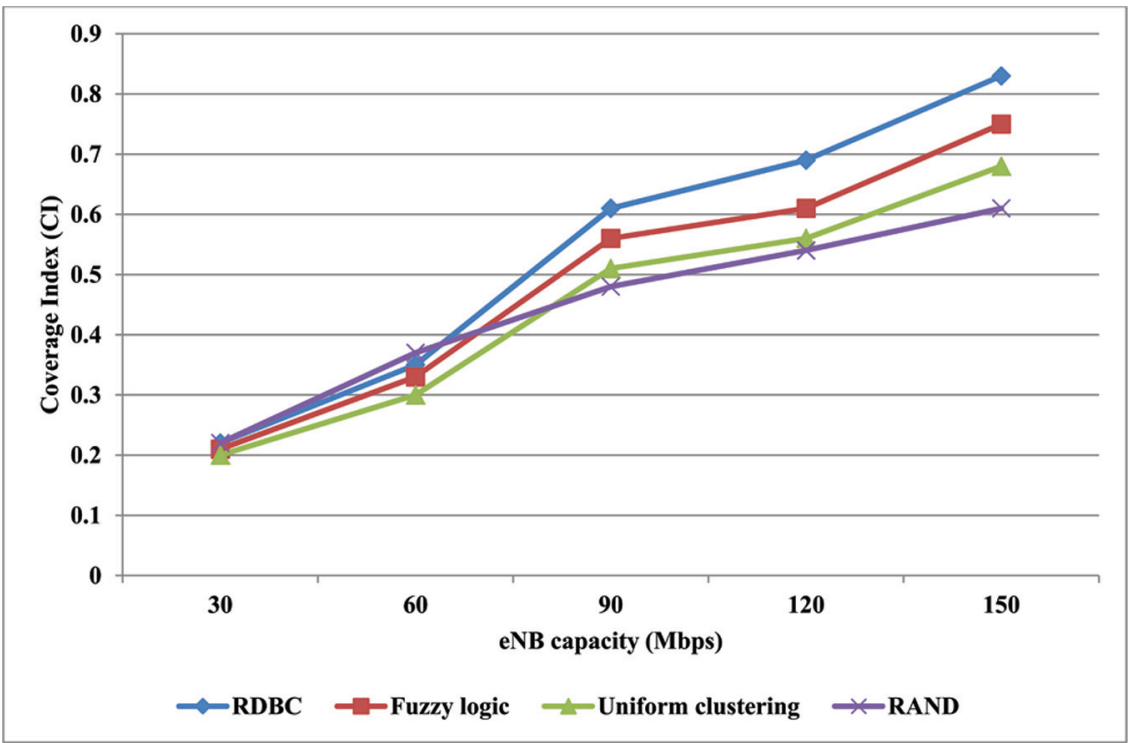

Figure 11 eNB capacity (Mbps) vs. CI comparison for different RS deployment schemes.

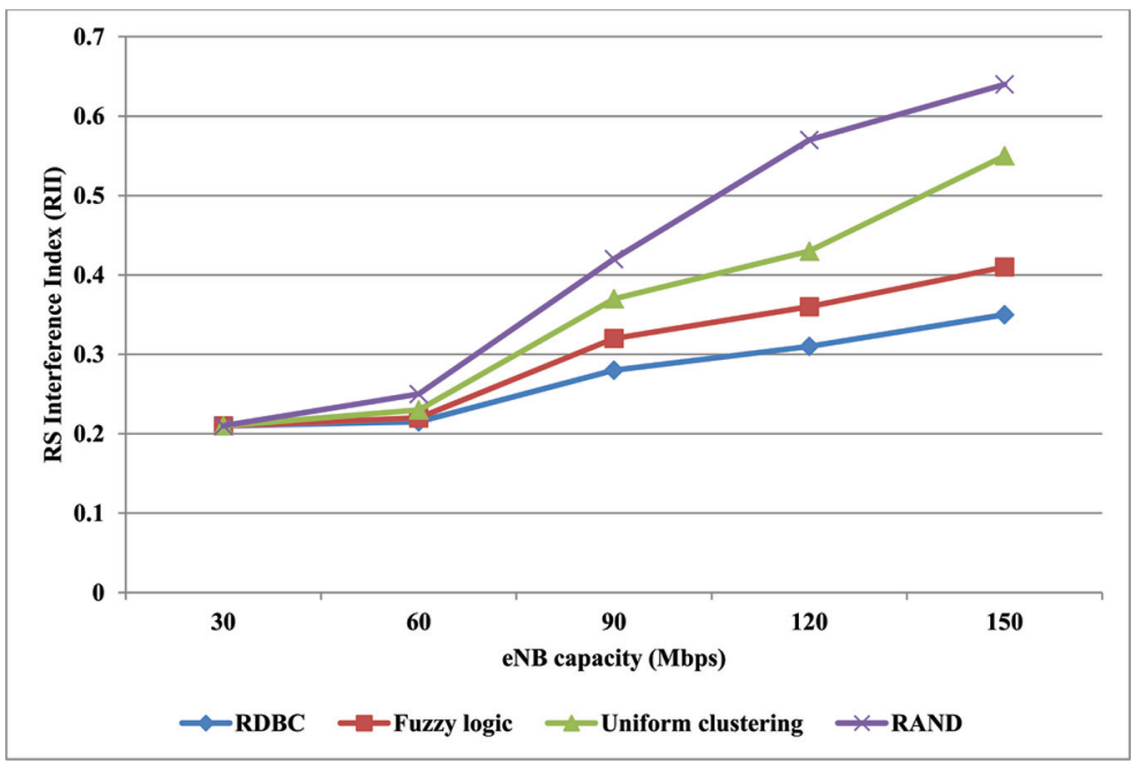

Figure 12 eNB capacity (Mbps) vs. RII comparison for different RS deployment schemes. 


\section{M. Arthi et al.}

to uniform clustering scheme and inferior to RDBC scheme. For an eNB capacity of $150 \mathrm{Mbps}$, our proposed RDBC scheme offers an improvement of approximately $45 \%$ over the conventional RAND scheme in terms of RII.

Figure 13 compares the PCEI impact factor of various deployment schemes for different values of eNB capacity. PCEI increases with the increase in eNB capacity irrespective of the deployment scheme. The proposed RDBC scheme offers better PCEI performance than the other conventional schemes. This is mainly due to the optimal identification of an RS deployment combination. The proposed scheme efficiently deploys the RSs so that the distance between the communicating stations and the corresponding path loss gets reduced. The MSs also effectively consume the transmitted power. The proposed scheme also uses NRS and MRS. The mobile nature of the serving nodes still reduces the distance between the communicating stations. This makes less transmission power sufficient to achieve the required data rate. The PCEI performance of the fuzzy logic based scheme is superior to uniform clustering scheme and inferior to RDBC scheme. For an eNB capacity of $150 \mathrm{Mbps}$, our proposed RDBC scheme offers an improvement of approximately $23 \%, 15 \%$ and $10 \%$ over the conventional RAND, uniform clustering and fuzzy logic based schemes respectively in terms of PCEI.

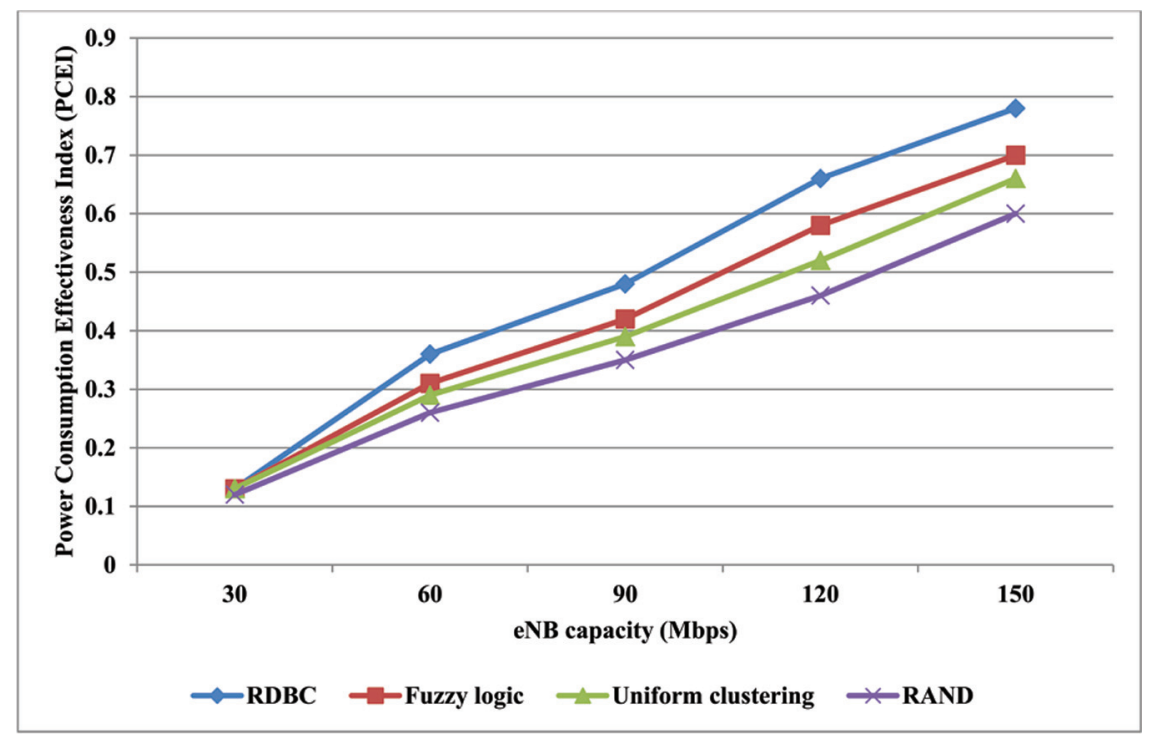

Figure 13 eNB capacity (Mbps) vs. PCEI comparison for different RS deployment schemes. 


\section{Conclusions}

In this work, a quality aware RS deployment scheme is proposed for the green radio communication. This work discusses different impact parameters and their importance in RS deployment. The simulation results show that the proposed scheme effectively identifies RS locations and their types based on the eNB capacity. The novel deployment leads to less transmission power allocation to users most of the time. The proposed scheme maximizes the transmission signal quality, coverage ratio and effectiveness in power consumption. Similarly, it minimizes the deployment cost and inter RS interference. The proposed scheme offers an average SSI, RPI, CI, RII and PCEI performances of $16 \%, 7 \%, 26 \%, 45 \%$ and $23 \%$ respectively over the conventional RAND scheme. Except in RPI, our proposed scheme offers improved performance than the fuzzy logic and uniform clustering based schemes.

The introduction of small cells like RSs, minimizes unnecessary deployment of eNBs. This novel scheme also minimizes $\mathrm{CO}_{2}$ emissions and environmental pollution. eNBs effectively offload the traffic to RSs. This also reduces the network load. In the present work, the MSs are assumed to be stationary. When the MSs are moving, there will be a significant degradation in the system performance. Framing a novel handoff algorithm is the future scope of this work.

\section{References}

[1] Alsharif, M. H., and Nordin, R., Ismail, M. (2013). Survey of green radio communications networks: techniques and recent advances. J. Comput. Netw. Commun. 2013, 13. doi: 10.1155/2013/453893

[2] Chen, T., Yang, Y., Zhang, H., Kim, H., and Horneman, K. (2011). Network energy saving technologies for green wireless access networks, IEEE Wirel. Commun. 18, 30-38.

[3] Ismail, M., and Zhuang, W. (2011). Network cooperation for energy saving in green radio communications, IEEE Wireless Commun. 18, $76-81$.

[4] Chang, B. J., Liang, Y. H., and Su, S. S. (2015). Analyses of Relay Nodes Deployment in 4G Wireless Mobile Multihop Relay Networks, Wirel. Pers. Commun. 83, 1159-1181.

[5] Rajendran, S., and Suriya, C. J. (2014). A Survey on energy efficient wireless communication in cellular networks. Int. J. Adv. Res. Comput. Eng. Technol. 11, 3609-3614. 
[6] Raab, F. H. (1987). Efficiency of Doherty RF power amplifier systems. IEEE Trans. Broadcast. 33, 77-83

[7] Niu, Z., Wu, Y., Gong, J., and Yang, Z. (2010). Cell zooming for costefficient green cellular networks. IEEE Commun. Mag. 48, 74-79.

[8] Balasubramanian, R. (2012). Cell zooming techniques for power efficient base station operation. San Deigo, CA: San Deigo State University.

[9] Lorincz, J., Capone, A., and Begusic, D. (2012). Impact of service rates and base station switching granularity on energy consumption of cellular networks, EURASIP J. Wirel. Commun. Netw. 1, 1-24.

[10] Ashraf, I., Boccardi, F., and Ho, L. (2011). Sleep mode techniques for small cell deployments. IEEE Commun. Mag. 49(8), 72-79.

[11] Wang, Y. C., and Chuang, C. A. (2015). Efficient eNB deployment strategy for heterogeneous cells in 4G LTE systems, Comput. Netw. 79, 297-312.

[12] Wu, J., Zhang, Y., Zukerman, M., and Yung, E. K. N. (2015). Energyefficient base-stations sleep-mode techniques in green cellular networks: a survey, IEEE Commun. Surv. Tuts. 17, 803-826.

[13] Feng, D., et al. (2013). A survey of energy-efficient wireless communications. IEEE Commun. Surv. Tuts., 15, 167-178.

[14] Chia,Y.-K., Sun, S., and Zhang, R. (2014). Energy cooperation in cellular networks with renewable powered base stations. IEEE Trans. Wireless Commun. 13, 6996-7010.

[15] Arthi, M., Arulmozhivarman, P., Vinoth Babu, K., Jose Joy, J., and Mariam George, E. (2015). Technical challenges in mobile multi-hop relay networks. Int. J. Appl. Eng. Res. 10, 26025-26036.

[16] Akyildiz, I. F., Gutierrez-estevez, D. M., Balakrishnan, R., Chavarriareyes, E. (2014). LTE Advanced and the evolution to Beyond 4G (B4G) systems. Phys. Commun. 10, 31-60 (2014).

[17] Arthi, M., Arulmozhivarman, P., and Vinoth Babu, K. (2015). Quality of service aware multi-hop relay networks for green radio communication. J. Green Eng. 5, 1-22.

[18] Yu, Y., Murphy, S. and, Murphy, L. (2008). "Planning base station and relay station locations in IEEE 802.16j multi-hop relay networks," in Proceedings of the Consumer communications and networking conference, 922-926.

[19] Kim, S. J., Kim, S. Y., Lee, B. B., Ryu, S. W., Lee, H. W., and Cho, C. H. (2008). "Multi-hop relay based coverage extension in the IEEE802.16j based mobile WiMAX systems," in Proceedings of the Fourth international conference on networked computing and advanced information management, Vol. 1, 516-522. 
[20] Ge, Y., Wen, S., and Ang, Y. H. (2009). "Analysis of optimal relay selection in IEEE 802.16 multi hop relay networks," in Proceedings of the IEEE wireless communications and networking conference, 1-6.

[21] Lu, H.-C., and Liao, W. (2009). "Joint base station and relay station placement for IEEE 802.16j networks," in Proceedings of IEEE global telecommunications conference (GLOBECOM), 1-5.

[22] Chang, J. Y. and Chen, Y. W. (2015). A cluster-based relay station deployment scheme for multi-hop relay networks. J. Commun. Netw. 17, 84-92.

[23] Vincent, M., Vinoth Babu, K., Arthi, M., and Arulmozhivarman, P. (2016). A novel fuzzy based relay node deployment scheme for multi-hop relay network, Proc. Technol. 24, 842-853.

[24] Tsourakis, T., and Voudouris, K. (2007). "WiMAX network planning and system's performance evaluation," in IEEE Wireless Communications and Networking Conference, 1948-1953.

[25] Shin, J. S., Kumar, R., Shin, Y. S., and La Porta, T. F. (2008). "Multi-hop wireless relay networks of mesh clients" in IEEE Wireless Communications and Networking Conference. 2717-2722.

[26] Chang, J. Y., and Lin, Y. S. (2015). An efficient base station and relay station placement scheme for multi-hop relay networks. Wirel. Pers. Commun. 82, 1907-1929.

[27] Chang, J. Y. and Lin, Y. S. (2014). A clustering deployment scheme for base stations and relay stations in multi-hop relay networks, Comput. Electr. Eng. 40, 407-420.

[28] Weisstein, E. Wolfram Mathworld. Available at: http://mathworld. wolfram.com/Circle-CircleIntersection.html

[29] European Telecommunications Standards Institute (2009-2007). LTE; Evolved Universal Terrestrial Radio Access (E-UTRA); Radio Frequency (RF) system scenarios (3GPP TR 36.942 version 8.2.0 Release 8). Technical Report, ETSI TR 136942 V8.2.0.

[30] European Telecommunications Standards Institute. (2011). LTE; Evolved Universal Terrestrial Radio Access (E-UTRA); Radio Frequency (RF) requirements for LTE Pico Node B. Technical Report, ETSI TR 136931 V9.0.0.

[31] 3rd Generation Partnership Project. (2011). Technical Specification Group Radio Access Network; Evolved Universal Terrestrial Radio Access (E-UTRA); Relay radio transmission and reception (Release 11), 3GPP TR 36.826 V11.0.0 (2012-09). 


\section{Biographies}

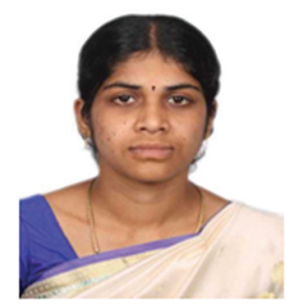

M. Arthi is presently working as a Research Associate in School of Electronics Engineering, VIT University, Vellore, Tamil Nadu, India. She has completed her B.E and M.E degrees from Anna University, Chennai, India in 2010 and 2012 respectively. Her area of interests includes Wireless networks and computer communication networks.

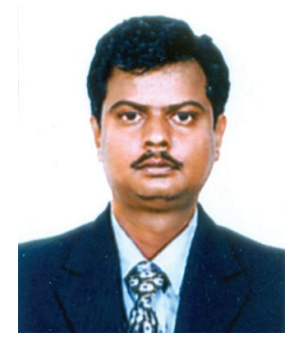

P. Arulmozhivarman was born in India in 1975. He received his Ph.D degree in the field of wavefront sensing and Adaptive optics from NIT Trichy, India in 2005 and he did both M.Sc., and B.Sc., Degree in Applied Physics (Instrumentation) in Bharathidasan University Trichy, and secured gold medal for both the programs. Currently he is working as a Professor in School of Electrical Engineering, Vellore Institute of Technology University (VIT), Vellore, India. Before joining the faculty of VIT, he was with NIT Trichy as a Senior Research Fellow in DRDO sponsored projects also served as Lecture in EIE Department in SASTRA University, Thanjavur during 1997-2000. He worked with DRDO scientists in the field of Imaging through atmospheric turbulence using Adaptive Optics system. He has successfully demonstrated the Shack Hartmann based wavefront sensor for adaptive Optics system at IRDE, DehraDun, DRDO Lab. He has successfully completed DRDO Sponsored project in the area of SAR Image Processing. He has successfully 
completed Collaborative research project for ADA Bangalore, Project titled, Design and Development of Optical Air data system for Advance Medium range Aircraft.

His research interest includes Biomedical Signal \& Image Processing, Vision based surveillance system, Biometric detection system and Image Compression and Machine Vision system for NDT evaluation and quality checking. Also he has undertaken DRDO Sponsored research projects in the area of remote sensing and image processing. He has produced $6 \mathrm{Ph}$.Ds and currently he is guiding $6 \mathrm{Ph} . \mathrm{D}$ candidates in the areas of Signal and Image processing and he has published more than 55 papers in National and International Journal. He served as one of the editor in several international conference proceedings and he is a reviewer of International Journal of Computer Engineering Research and IEEE Photonics Technology letters. Dr. P. Arulmozhivarman is a member of IEEE signal processing society.

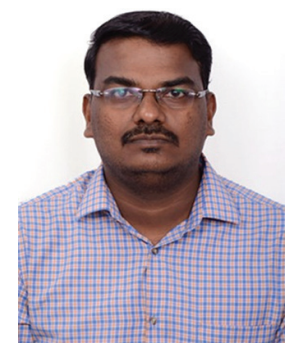

K. Vinoth Babu is presently working as an Associate Professor in School of Electronics Engineering, VIT University, Vellore, Tamil Nadu, India. He has completed his Ph.D and M.Tech degrees from VIT University, Vellore, India in 2014 and 2009 respectively and B.E (ECE) Degree from Anna University, Chennai, India in 2005. His area of interests includes Wireless Digital Communications and Signal Processing. 
